\title{
Análise da Influência de Fatores Externos Associados à Avaliação da Qualidade do Ensino Online
}

\author{
Germano O. Ribeiro ${ }^{1}$, Thomaz E.V. Silva ${ }^{2}$, Albano O. Nunes ${ }^{3}$, \\ F. Herbert L. Vasconcelos ${ }^{1}$ \\ ${ }^{1}$ Instituto UFC Virtual - Universidade Federal do Ceará \\ ${ }^{2}$ Departmento de Engenharia de Teleinformática - Universidade Federal do Ceará \\ Campus do Pici - Fortaleza - Ceará - Brasil. \\ ${ }^{3}$ Faculdade Vale do Jaguaribe -FVJ \\ Aracati - Ceará - Brasil. \\ germanoribeirolo@gmail.com, thomazvelosodvirtual.ufc.br, \\ albanodfvj.br, herbertevirtual.ufc.br
}

\begin{abstract}
This research aims to evaluate a new indicator of quality of education and the relationship of the indicators assessed with two external factors evaluation. The study was conducted in continuing education courses in a Federal Institution of Higher Education. The results showed that the autonomy indicator still needs to be improved with actions by the students in order to encourage them to develop that autonomy. Another result was the finding that gender differences and the amount of previously distance education courses undertaken by students had not influenced the assessment of quality of education.
\end{abstract}

Resumo. Esta pesquisa tem como objetivo avaliar um novo indicador de qualidade do ensino e a relação dos indicadores avaliados com dois fatores externos à avaliação. O estudo foi conduzido em cursos de formação continuada de uma Instituição Federal de Ensino Superior. Os resultados apontam que o indicador Autonomia ainda precisa ser melhorado com ações junto ao cursista, de modo a estimulá-lo no desenvolvimento dessa autonomia. Outro resultado obtido foi a constatação de que as diferenças de gênero e da quantidade de cursos em EaD já realizados pelos cursistas previamente, não influenciam a avaliação da qualidade do ensino.

\section{Introdução}

A partir da democratização do acesso à educação superior à distância no início do século XXI, a participação dos estudantes nessa modalidade de ensino vem tornando-se cada vez mais representativa CensoEAD.BR (2013). Já com a consolidação do interesse da comunidade acadêmica na utilização das Tecnologias Digitais da Informação e Comunicação (TDIC), um dos grandes desafios dessa utilização é a manutenção de uma boa qualidade do ensino.

Acompanhando a expansão da Educação à Distância $(\mathrm{EaD})$, a produção de instrumentos e metodologias de avaliação se faz cada vez mais necessários para a verificação da boa qualidade de aprendizagem de cursos ofertados por esta modalidade de ensino, fazendo com que pesquisadores e agências financiadoras direcionem estudos e recursos para a investigação dessa temática [Attwell 2006]. 
No ensino superior presencial, o aumento da demanda por cursos com uma boa qualidade de ensino, associados a boas práticas de gestão educacionais, tornaram-se fonte de investigação de diversos pesquisadores [Howard, Conway, e Maxwell 1985; Urbina, Pérez e Rodríguez 2013; Nunes 2015]. O desenvolvimento e validação de instrumentos de avaliação que contemplem o aumento da demanda supracitada faz com que mais estudantes universitários sejam chamados a participar de avaliações institucionais acerca da qualidade do ensino de seus respectivos cursos [Marsh e Bailey 1993].

A avaliação da qualidade do ensino, proposta inicialmente por Marsh e Bailey (1993) e, posteriormente, adaptada para a EaD brasileira, a partir do formato da Universidade Aberta do Brasil [Ribeiro et al 2013], será levada em consideração para a avaliação de três cursos ofertados por uma Instituição Federal de Ensino Superior. Torna-se importante ressaltar que a pesquisa conduzida por Ribeiro et al (2014) apresenta resultados satisfatórios no que se refere a redução das taxas de evasão, a partir da análise dos indicadores de qualidade do ensino e intervenção gerencial pós-avaliação. Nesta pesquisa, além da utilização do instrumento de avaliação previamente proposto por Ribeiro et al (2013), há uma análise de questões externas aos indicadores de qualidade, como por exemplo o gênero dos cursistas e a quantidade de cursos já realizado pelos mesmos na modalidade EaD. Dessa forma, este artigo apresenta as seguintes contribuições:

- O incremento do fator Autonomia no instrumento de avaliação proposto por Ribeiro et al (2013).

- A análise, a partir da utilização da ANOVA One-Way, da influência do gênero e da experiência dos cursistas que já participaram previamente de outros cursos na modalidade $\mathrm{EaD}$ na avaliação da qualidade do ensino.

Este estudo está dividido em cinco partes: inicialmente, a qualidade dos cursos em EaD serão discutidas, bem como sua importância para a melhoria do ensino no Brasil; Uma breve explanação sobre a Análise de Variância (ANOVA) será dada, no intuito de esclarecer o porque esta técnica foi utilizada para analisar os dados obtidos pela pesquisa. No momento referente a metodologia, serão apresentadas as assertivas que compõem a capacidade técnica dos respondentes em avaliar o curso semipresencial, e o seu campo amostral. No tópico destinado aos resultados da pesquisa, os dados coletados e os respectivos testes estatísticos utilizados para comparar a capacidade técnica dos respondentes com outros fatores do instrumento avaliativo serão analisados. E nas conclusões estarão expostas as limitações da pesquisa, suas potencialidades e perspectivas futuras.

\section{Avaliação da Qualidade de Cursos em EaD}

Neste tópico do texto, explana-se a respeito da qualidade na educação à distância e da importância de se elencar e avaliar eficazmente fatores relacionados ao ensino semipresencial para desenvolvimento de indicadores que retratem com fidelidade a qualidade desta modalidade de ensino.

Segundo Sobrinho (2011), a avaliação deve ser um empreendimento coletivo que busque compreender os sentidos múltiplos, e até contraditórios, dos processos 
relacionais produzidos na Universidade. A compreensão de tais aspectos nos leva a crer que a qualidade educativa, além de uma construção social, deve ser compreendida como um processo de caráter essencialmente pedagógico que evite a exposição pública das limitações individuais dos envolvidos nas análises.

Transpondo para o Ensino à Distância algumas das questões que cercam a avaliação das instituições, percebe-se que analisar a qualidade dos cursos à distância é bastante complexo, em virtude desta modalidade de ensino ser caracterizada por uma separação espacial e temporal que ganham contornos muito específicos e necessitam de uma percepção mais acurada dos diversos papeis desempenhados por aqueles envolvidos no Ensino à Distância.

Em Lachi et al, (2006); Rocha et al, (2011); Ribeiro et al, (2013) e Silva et al, (2015) percebe-se um pouco desta complexidade. Nestas pesquisas há a apresentação e discussão de questões sobre as estratégias de extração de informação, e delimitação de efeitos da organização do processo pedagógico-administrativo na dinâmica das Universidades, sendo possível também a proposição de novas abordagens e intervenções administrativas que possam promover a melhoria da qualidade do ensino online.

O processo de avaliação do ensino à distância é visto, dentre outros aspectos, como a possibilidade de cada instituição obter um diagnóstico do cenário e ter subsídios para definir quais critérios/dimensões são prioridades para o aprimoramento desses processos e traçar estratégias de modo a efetivar a aprendizagem de seus alunos. Esta visão a respeito da avaliação vem servindo de trilha para uma mudança geral de comportamentos, saindo da simples mensuração de conteúdo cognitivos para o quanto a instituição colaborou com desenvolvimento das capacidades de seus aprendizes.

\section{Análise de Variância One-Way (ANOVA)}

De uma forma bem geral, a Análise de Variância One-Way (ANOVA) é utilizada para determinar se existe alguma diferença entre as médias de três, ou mais, fatores/grupos independentes [Gelman 2005]. A ANOVA compara as médias entre os grupos de interesse e determina se as médias obtidas são, ou não, estatisticamente significantes. Especificamente, o modelo testa a seguinte hipótese nula:

$$
H_{0}: \mu_{1}=\mu_{2}=\cdots=\mu_{k}=\cdots=\mu_{K} \text {, }
$$

onde $\mu_{k}(\forall k=1,2,3, \ldots, K)$ representa a média do $k$-ésimo grupo e $K$ representa o número de grupos analisados. No entanto, se a ANOVA retornar valores significativos entre as médias, então se aceita a hipótese alternativa $\left(H_{1}\right)$ de que existam, no mínimo, dois grupos que são significativamente diferentes entre si.

Três pressupostos probabilísticos precisam ser levados em consideração para a utilização da ANOVA, são eles segundo Gelman (2005):

1. Observações Independentes - todas as observações analisadas são coletadas de forma independente;

2. Normalidade - a distribuição do erro das medidas segue uma distribuição normal;

3. Homogeneidade das Variâncias - assume-se que as variâncias dos grupos são homogêneas, ou seja, seguem a mesma distribuição estatística. 
V Congresso Brasileiro de Informática na Educação (CBIE 2016)

Anais do XXII Workshop de Informática na Escola (WIE 2016)

Todos os procedimentos para o cálculo e análise da ANOVA são descritas em Bailey (2008).

\section{Metodologia}

Nesta seção serão apresentados os procedimentos de coleta de dados da amostra pesquisada, sua caracterização e a estrutura do instrumento avaliativo utilizado para coletar a resposta dos cursistas.

\subsection{Caracterizando a Amostra}

O campo amostral desta pesquisa é composto por três (03) turmas de formação docente semipresencial nas áreas de Gênero e Diversidade na Escola (GDE), Educação de Jovens e Adultos (EJA), Escola Sustentáveis e Com-Vidas (ESCV), Direitos Humanos (DH) e Educação Ambiental (EA). Os cursos de extensão possuem entre 180 a 200 horas/aulas distribuídos em módulos, tendo duração média de quatro meses. Os cursos são compostos por momentos presenciais e a distância, mediados por um Ambiente Virtual de Aprendizagem (AVA), por meio dos quais os cursistas são estimulados a identificar um problema concreto em sua comunidade escolar e em seguida propor uma intervenção pedagógico-administrativa por meio de um projeto.

No ano de 2014, um total de 1639 professores foram certificados pelos referidos cursos de formação continuada. Todos os professores concludentes estavam aptos a participarem desta pesquisa, visando contribuir com sua percepção do curso, para melhoria da qualidade das futuras ofertas.

Ao final de cada curso, os professores certificados foram convidados, a partir do correio eletrônico individual, a responder o questionário disponibilizado online. A amostra obtida consistiu apenas daqueles respondentes que, efetivamente, responderam de forma completa a pesquisa. Assim sendo, para a análise dos resultados, foram considerados os dados fornecidos por 791 professores concludentes dos cursos, ou seja, $48,5 \%$ do público alvo total.

Tabela 1 - Faixa Etária dos Cursistas.

\begin{tabular}{|c|c|c|c|c|c|}
\hline & & Frequência & Porcentual & $\begin{array}{c}\text { Porcentagem } \\
\text { válida }\end{array}$ & $\begin{array}{l}\text { Porcentagem } \\
\text { acumulativa }\end{array}$ \\
\hline \multirow{6}{*}{ Válido } & $20-30$ anos & 195 & 24,7 & 24,7 & 24,7 \\
\hline & $30-40$ anos & 274 & 34,6 & 34,6 & 59,3 \\
\hline & $40-50$ anos & 217 & 27,4 & 27,4 & 86,7 \\
\hline & $50-60$ anos & 96 & 12,1 & 12,1 & 98,9 \\
\hline & $\begin{array}{l}\text { Acima de } 60 \\
\text { anos }\end{array}$ & 9 & 1,1 & 1,1 & 100 \\
\hline & Total & 791 & 100 & 100 & \\
\hline
\end{tabular}

Tabela 2 - Formação Acadêmica dos Cursistas

\begin{tabular}{|l|r|r|r|r|}
\hline & Frequência & Porcentual & $\begin{array}{c}\text { Porcentagem } \\
\text { válida }\end{array}$ & $\begin{array}{c}\text { Porcentagem } \\
\text { acumulativa }\end{array}$ \\
\hline Bacharel & 55 & 7 & 7 & 7 \\
Licenciado em Ciências da Natureza & 174 & 22 & 22 & 29 \\
Licenciado em Ciências da Saúde & 9 & 1,1 & 1,1 & 30,1 \\
Válido & 308 & 38,9 & 38,9 & 69 \\
Licenciado em Ciências Humanas & 245 & 31 & 31 & 100 \\
Licenciado em Linguagem e & 791 & 100 & 100 & \\
Códigos & & & &
\end{tabular}


V Congresso Brasileiro de Informática na Educação (CBIE 2016)

Anais do XXII Workshop de Informática na Escola (WIE 2016)

$\mathrm{Na}$ Tabela 1, pode-se observar que a maioria dos cursistas tem idade entre 20 e 50 anos $(86,7 \%)$, e esse resultado indica que os professores que ainda estão em pleno desenvolvimento de suas atividades e funções estão à procura de formações continuadas a fim de aprimorar o seu próprio conhecimento.

Outro ponto interessante a ser considerado é o resultado apresentado na Tabela 2. Observando as características multidisciplinares dos cursos ofertados, não há a predominância de uma área do conhecimento específica para aqueles que atenderam os cursos, sendo os cursos em Licenciatura aqueles que tiveram maior incidência.

\subsection{Dinâmica da pesquisa.}

O instrumento de coleta de dados utilizado como base para essa pesquisa foi o proposto por Ribeiro et al, (2013) e aprimorado por Silva et al, (2015). O instrumento original conta com 29 assertivas distribuídas em cinco indicadores de qualidade do ensino (ver Figura 1). A inclusão de mais cinco assertivas, totalizando 34, se deu pela necessidade de se avaliar o grau de autonomia dos cursistas e para entender a relação desse fator com os demais indicadores de qualidade do ensino. Para responder o instrumento avaliativo, o cursista necessitava atribuir um grau de concordância a cada uma das assertivas utilizando uma escala Likert de cinco pontos (1- Discordo Fortemente, 2- Discordo parcialmente, 3- Indiferente, 4- Concordo parcialmente até 5Concordo Fortemente).

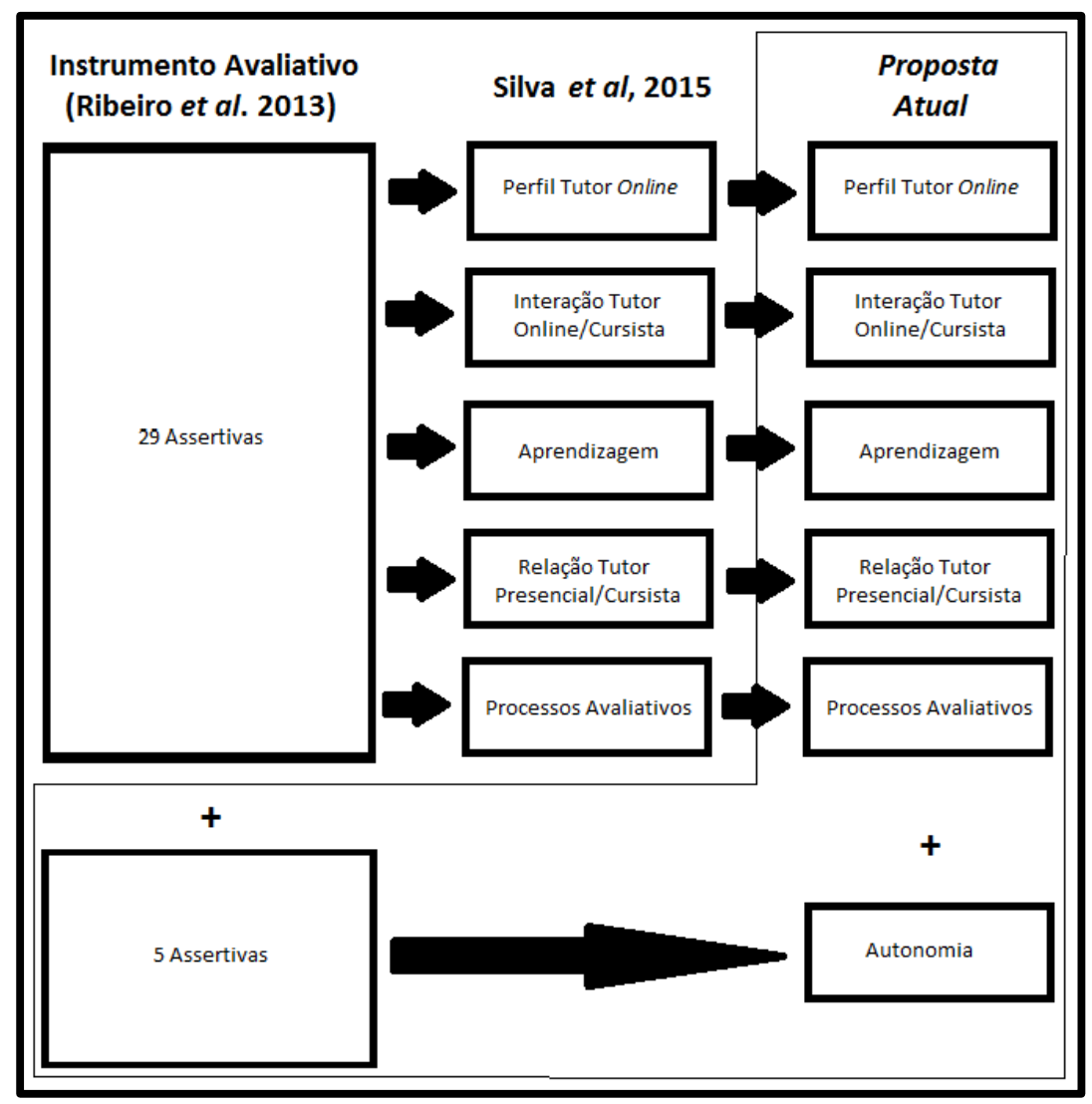

Figura 1 - Acrescentando o fator Autonomia no modelo proposto por Ribeiro et al. (2013).

O instrumento avaliativo ficou disponível para os cursistas por 20 dias após o término dos cursos e o envio das respostas era realizado de forma automática. Por 
V Congresso Brasileiro de Informática na Educação (CBIE 2016)

Anais do XXII Workshop de Informática na Escola (WIE 2016)

questão de espaço o instrumento avaliativo não será apresentado, mas pode ser solicitado a um dos autores via correio eletrônico.

Todos os dados e resultados da pesquisa foram tabulados e extraídos com a utilização do software SPSS ${ }^{\circledR}$.

\section{Resultados Obtidos}

\subsection{Estatística Descritiva}

Nesta seção, cada um dos seis indicadores de qualidade do ensino foi avaliado quanto as suas médias e desvios padrão. Como pode ser observado na Tabela 3, o indicador Interação Tutor Online/Cursista foi o melhor avaliado pelos cursistas. Dentre os cinco primeiros indicadores apresentados, o pior avaliado foi o indicador Relação Tutor Presencial/Cursista. Esses dois resultados já eram esperados, tendo em vista que algumas políticas de contato com o cursista foram utilizadas ao longo do curso, no intuito de diminuir as possíveis taxas de evasão e todos os contatos eram feitos pelos tutores online. Em alguns polos não houve a figura do tutor presencial por conta da falta de professores capacitados nas áreas dos cursos para atuar na localidade do polo, o que pode ter influenciado diretamente a avaliação negativa do indicador, principalmente pelo alto desvio padrão observado.

Tabela 3 - Estatística Descritiva dos Fatores do Instrumento Avaliativo.

\begin{tabular}{|c|c|c|c|c|c|}
\hline & $\mathrm{N}$ & Mínimo & Máximo & Média & $\begin{array}{l}\text { Desvio } \\
\text { padrão }\end{array}$ \\
\hline Aprendizagem & 791 & 1,5 & 5 & 4,6391 & 0,43369 \\
\hline Perfil do Tutor Online & 791 & 1 & 5 & 4,6124 & 0,64126 \\
\hline $\begin{array}{l}\text { Interação Tutor } \\
\text { Online/Cursista }\end{array}$ & 791 & 1 & 5 & 4,6982 & 0,57492 \\
\hline $\begin{array}{l}\text { Relação Tutor } \\
\text { Presencial/Cursista }\end{array}$ & 791 & 1 & 5 & 4,5066 & 0,7663 \\
\hline Processos Avaliativos & 791 & 1 & 5 & 4,5626 & 0,5752 \\
\hline Autonomia & 791 & 1,8 & 5 & 4,4326 & 0,51292 \\
\hline
\end{tabular}

Desde o desenvolvimento do instrumento avaliativo proposto por Ribeiro et al. (2013), pela primeira vez o indicador Autonomia foi avaliado e pode-se observar que ele obteve o pior desempenho dentre os demais. Esse indicador pode ser relacionado como um exame de autoavaliação do cursista a luz do seu comportamento autônomo. $\mathrm{O}$ baixo escore atribuído a esse indicador ressalta a necessidade de políticas direcionadas a aquisição de autonomia por parte dos cursistas, esta fragilidade pode ainda ser refletida no alto valor atribuído ao tutor online, já que este atuava como elemento motivador durante todo o curso.

\subsection{Análise da Correlação entre os Indicadores de Qualidade do Ensino}

Nesta seção, o coeficiente de correlação de Pearson para cada um par de variáveis foi computado e apresentado na Tabela 4. Todos os níveis de significância ficaram abaixo de .01, garantindo a significância dos coeficientes.

O coeficiente de correlação indica a dependência estatística das distribuições entre as variáveis analisadas [Gelman 2005]. Dessa forma, pode ser destacado uma alta correlação (.801) entre os indicadores relacionados ao tutor online, o que faz sentido já que os cursistas estão avaliando a mesma pessoa e que a partir do perfil do tutor pode-se ter uma noção de como será sua interação no AVA. 
V Congresso Brasileiro de Informática na Educação (CBIE 2016)

Anais do XXII Workshop de Informática na Escola (WIE 2016)

Tabela 4 - Matriz de Correlação* dos Fatores Avaliados.

\begin{tabular}{|c|c|c|c|c|c|c|c|}
\hline & & Aprendizagem & $\begin{array}{l}\text { Perfil } \\
\text { do } \\
\text { Tutor } \\
\text { Online }\end{array}$ & $\begin{array}{c}\text { Interação } \\
\text { Tutor } \\
\text { Online/ } \\
\text { Cursista }\end{array}$ & $\begin{array}{l}\text { Relação } \\
\text { Tutor } \\
\text { Presencial/ } \\
\text { Cursista }\end{array}$ & $\begin{array}{l}\text { Processos } \\
\text { Avaliativos }\end{array}$ & Autonomia \\
\hline Aprendizagem & $\begin{array}{l}\text { Correlação } \\
\text { de Pearson } \\
\text { Sig. }(\rho)\end{array}$ & 1 & & & & & \\
\hline $\begin{array}{l}\text { Perfil do Tutor } \\
\text { Online }\end{array}$ & $\begin{array}{l}\text { Correlação } \\
\text { de Pearson } \\
\text { Sig. }(\rho)\end{array}$ & $\begin{array}{r}, 620 \\
0\end{array}$ & 1 & & & & \\
\hline \begin{tabular}{|l|} 
Interação \\
Tutor Online/ \\
Cursista
\end{tabular} & $\begin{array}{l}\text { Correlação } \\
\text { de Pearson } \\
\text { Sig. }(\rho)\end{array}$ & $\begin{array}{r}, 564 \\
0\end{array}$ & $\begin{array}{r}, 801 \\
0\end{array}$ & 1 & & & \\
\hline \begin{tabular}{|l|} 
Relação Tutor \\
Presencial/ \\
Cursista
\end{tabular} & $\begin{array}{l}\text { Correlação } \\
\text { de Pearson } \\
\text { Sig. }(\rho)\end{array}$ & $\begin{array}{r}, 350 \\
0\end{array}$ & $\begin{array}{r}, 541 \\
0\end{array}$ & $\begin{array}{r}, 468 \\
0\end{array}$ & 1 & & \\
\hline $\begin{array}{l}\text { Processos } \\
\text { Avaliativos }\end{array}$ & $\begin{array}{l}\text { Correlação } \\
\text { de Pearson } \\
\text { Sig. }(\rho)\end{array}$ & $\begin{array}{r}, 626 \\
0\end{array}$ & $\begin{array}{r}, 737 \\
0\end{array}$ & $\begin{array}{r}669 \\
0\end{array}$ & $\begin{array}{r}, 482 \\
0\end{array}$ & 1 & \\
\hline Autonomia & $\begin{array}{l}\text { Correlação } \\
\text { de Pearson } \\
\text { Sig. }(\rho)\end{array}$ & $\begin{array}{r}, 399 \\
0\end{array}$ & $\begin{array}{r}, 324 \\
0\end{array}$ & ,319 & $\begin{array}{r}, 242 \\
0\end{array}$ & $\begin{array}{r}, 428 \\
0\end{array}$ & 1 \\
\hline
\end{tabular}

*Todos os coeficientes de correlação apresentaram significância estatística de $\rho<.01$.

A correlação do indicador Autonomia com os indicadores Aprendizagem, Perfil do Tutor Online, Interação Tutor Online/Cursista e Relação Tutor Presencial/Cursista pode ser considerada baixa. Esse resultado reflete a não influencia desse indicador em questões que não dizem respeito a sua autonomia, reforçando que esta autonomia é algo particular do cursista não possuindo relação direta com os fatores em análise. Entretanto, com o fator Processos Avaliativos há uma correlação moderada (.428), deve-se então considerar que as assertivas relacionadas a esse indicador apontam ao nível de estudo/dedicação dos cursistas para com os processos avaliativos do curso que, de certa forma, vai também estar associada a autonomia do cursista.

\subsection{ANOVA}

Conforme apresentado na Seção 3, a ANOVA determina se há, ou não, significância estatística entre as médias dos indicadores analisados. Sendo assim, para esse estudo, duas hipóteses nulas serão consideradas:

1. Não há significância estatística da avaliação realizada pelos cursistas que já haviam participado de outros cursos na modalidade EaD para aqueles que estão realizando um curso nessa modalidade pela primeira vez; e

2. Não há significância estatística da avaliação realizada por cursistas de gêneros diferentes.

Sendo assim, as variáveis independentes consideradas neste estudo são: Número de Cursos em EaD que o Cursista já participou e os Gêneros dos cursistas. As variáveis dependentes são os indicadores de qualidade do ensino extraídos com a aplicação do instrumento avaliativo.

A definição do "número de cursos em $\mathrm{EaD}$ que os cursistas já haviam participado" como variáveis independentes partiu do senso comum relacionado ao fato do cursista que tem mais "experiência" em cursos na modalidade EaD são mais criteriosos na hora de avaliar um curso, pois ele já teria como parâmetro outros cursos. A variável independente "Gênero" foi escolhida com base em trabalhos que 
V Congresso Brasileiro de Informática na Educação (CBIE 2016)

Anais do XXII Workshop de Informática na Escola (WIE 2016)

encontraram diferenças significativas nas avaliações quando compararam o gênero dos avaliadores [Nicholls 1975; Dweck e Bush 1976].

Tabela 5 - ANOVA Aplicada a Variável "Número de Cursos em EaD que o Cursista já Participou".

\begin{tabular}{|ll|r|r|r|c|c|}
\hline & & $\begin{array}{r}\text { Soma dos } \\
\text { Quadrados }\end{array}$ & df & $\begin{array}{c}\text { Quadrado } \\
\text { Médio }\end{array}$ & F & Sig. \\
\hline \multirow{2}{*}{ Aprendizagem } & Entre Grupos & 0,528 & 4 & 0,132 & 0,701 & 0,591 \\
& Nos grupos & 148,064 & 786 & 0,188 & & \\
& Total & 148,592 & 790 & & & \\
Perfil do Tutor & Entre Grupos & 3,071 & 4 & 0,768 & 1,876 & 0,113 \\
Online & Nos grupos & 321,787 & 786 & 0,409 & & \\
& Total & 324,859 & 790 & & & \\
Interação Tutor & Entre Grupos & 1,324 & 4 & 0,331 & 1,002 & 0,406 \\
Online/Cursista & Nos grupos & 259,801 & 786 & 0,331 & & \\
Relação & Total & 261,125 & 790 & & & \\
Presencial/Cursistar & Entre Grupos & 4,893 & 4 & 1,223 & 2,095 & 0,08 \\
& Nos grupos & 459,009 & 786 & 0,584 & & \\
Processos & Total & 463,903 & 790 & & & \\
Avaliativos & Entre Grupos & 1,599 & 4 & 0,4 & 1,209 & 0,305 \\
& Nos grupos & 259,774 & 786 & 0,331 & & \\
Autonomia & Total & 261,372 & 790 & & & \\
& Entre Grupos & 0,707 & 4 & 0,177 & 0,67 & 0,613 \\
& Nos grupos & 207,132 & 786 & 0,264 & & \\
\hline
\end{tabular}

As Tabelas 5 e 6 apresentam os resultados para ambas as variáveis independentes em relação às variáveis dependentes. Percebe-se que, em ambos os casos, os níveis de significância não foram obtidos $(\rho<.01$.).

Tabela 6 - ANOVA Aplicada a Diferença de Gênero.

\begin{tabular}{|c|c|c|c|c|c|c|}
\hline & & \begin{tabular}{|l} 
Soma dos \\
Quadrados \\
\end{tabular} & $d f$ & $\begin{array}{l}\text { Quadrado } \\
\text { Médio }\end{array}$ & $F$ & Sig. \\
\hline Aprendizagem & $\begin{array}{l}\text { Entre Grupos } \\
\text { Nos grupos } \\
\text { Total }\end{array}$ & $\begin{array}{r}0,119 \\
148,473 \\
148,592\end{array}$ & $\begin{array}{r}1 \\
789 \\
790\end{array}$ & $\begin{array}{l}0,119 \\
0,188\end{array}$ & 0,632 & 0,427 \\
\hline $\begin{array}{l}\text { Perfil do Tutor } \\
\text { Online }\end{array}$ & $\begin{array}{l}\text { Entre Grupos } \\
\text { Nos grupos } \\
\text { Total }\end{array}$ & $\begin{array}{r}0,289 \\
324,569 \\
324,859\end{array}$ & $\begin{array}{r}1 \\
789 \\
790\end{array}$ & $\begin{array}{l}0,289 \\
0,411\end{array}$ & 0,703 & 0,402 \\
\hline $\begin{array}{l}\text { Interação Tutor } \\
\text { Online/Cursista }\end{array}$ & $\begin{array}{l}\text { Entre Grupos } \\
\text { Nos grupos } \\
\text { Total }\end{array}$ & $\begin{array}{r}0,813 \\
260,312 \\
261,125\end{array}$ & $\begin{array}{r}1 \\
789 \\
790\end{array}$ & $\begin{array}{r}0,813 \\
0,33\end{array}$ & 2,465 & 0,117 \\
\hline $\begin{array}{l}\text { Relação Tutor } \\
\text { Presencial/Cursista }\end{array}$ & $\begin{array}{l}\text { Entre Grupos } \\
\text { Nos grupos } \\
\text { Total }\end{array}$ & $\begin{array}{r}0,036 \\
463,867 \\
463,903\end{array}$ & $\begin{array}{r}1 \\
789 \\
790\end{array}$ & $\begin{array}{l}0,036 \\
0,588\end{array}$ & 0,061 & 0,804 \\
\hline $\begin{array}{l}\text { Processos } \\
\text { Avaliativos }\end{array}$ & $\begin{array}{l}\text { Entre Grupos } \\
\text { Nos grupos } \\
\text { Total }\end{array}$ & $\begin{array}{r}0,002 \\
261,37 \\
261,372\end{array}$ & $\begin{array}{r}1 \\
789 \\
790 \\
\end{array}$ & $\begin{array}{l}0,002 \\
0,331\end{array}$ & 0,007 & 0,933 \\
\hline Expertise & $\begin{array}{l}\text { Entre Grupos } \\
\text { Nos grupos } \\
\text { Total }\end{array}$ & $\begin{array}{r}0 \\
207,838 \\
207,838\end{array}$ & $\begin{array}{r}1 \\
789 \\
790\end{array}$ & $\begin{array}{r}0 \\
0,263\end{array}$ & 0,002 & 0,967 \\
\hline
\end{tabular}

Dessa forma, com base nos resultados obtidos podemos afirmar que não há nenhuma relação das avaliações proferidas por cursistas que já haviam participado de outros cursos em EaD para aqueles que estavam participando pela primeira vez. $\mathrm{E}$ também não há distinção na avaliação em relação à diferença de gênero dos cursistas. Sendo assim, em ambos as situações consideradas, a hipótese nula foi confirmada. 
V Congresso Brasileiro de Informática na Educação (CBIE 2016)

Anais do XXII Workshop de Informática na Escola (WIE 2016)

\section{Considerações Finais}

Estre trabalho propôs a contribuição de dois aspectos: 1. A inclusão do indicador Autonomia dentre os indicadores propostos por Ribeiro et al. (2015), e 2. Analisar os indicadores de qualidade do ensino sob a ótica da influência de dois fatores externos: A quantidade de cursos em EaD já realizado pelos cursistas e o gênero dos mesmos.

$\mathrm{O}$ indicador Autonomia apresentou um baixo valor de média, quando comparado aos outros indicadores, o que indica que os cursistas não tinham autonomia plena quanto à autocondução das suas atividades online. Esse mesmo indicador apresentou, em sua maioria, correlações baixas com os demais indicadores, apresentando correlação moderada apenas com o indicador Processos Avaliativos, o qual, de certa forma, está intrinsicamente relacionado com a autonomia do cursista.

Os resultados apontaram para a não relação entre os indicadores de qualidade do ensino e as duas variáveis independentes consideradas. Esse resultado desmistifica o senso comum de que o cursista que já participou de cursos na modalidade EaD tornamse mais criteriosos na avaliação do que aqueles que estão participando do curso nessa modalidade pela primeira vez.

Alguns cuidados precisam ser levados em consideração na aplicação do instrumento avaliativo. A análise cega e sem levar em consideração o contexto da avaliação pode fazer com que os gestores do processo avaliado tomem atitudes equivocadas no intuito de melhorar a qualidade do ensino. Um estudo mais amplo e uma compreensão mais apurada do contexto são fundamentais para a análise correta dos indicadores de qualidade do ensino.

\section{Referências Bibliográficas}

Attwell, G. (2006) "Evaluating e-learning: a guide to the evaluation of the e-learning". Evaluate Europe Handbook Series Volume 2, European Commission.

Bailey, R. A. (2008) "Design of Comparative Experiments". Cambridge University Press.

Censo EAD.BR. (2013) "Relatório Analítico da Aprendizagem a Distância no Brasil 2012". Curitiba: Ibpex.

Dweck, C. S., e Bush, E. S. (1976) "Sex differences in learned helplessness: I. Differential debilitation with peer and adult evaluators". Developmental Psychology, n. 12 , p. 147-156.

Galvão, A., Perfeito, C. e Macedo, R. (2011) "Desenvolvimento de expertise: um estudo de caso". Revista Diálogo Educacional, Curitiba, v.11, n.34, p.1015-1033, set/dez.

Gelman, A. (2005) "Analysis of variance? Why it is more important than ever". The Annals of Statistics. n. 33, p. 1-53.

Howard, G. S.; Conway, C. G.; Maxwell, S. E. (1985) "Construct validity of measures of college teaching effectiveness", Journal of Educational Psychology, vol.77, n.2, pp. 187-196.

Lachi, R. L., Rocha, H. V. e Oeiras, J. Y. Y. (2006) "Avaliação de cursos à distância: uso de indicadores para assegurar qualidade". Anais do XVII SBIE. 
V Congresso Brasileiro de Informática na Educação (CBIE 2016)

Anais do XXII Workshop de Informática na Escola (WIE 2016)

Marsh, H. W. e Bailey, M. (1993) 'Multidimensional students' evaluations of teaching effectiveness: A profile analysis". The Journal of Higher Education, Vol. 64: p. 1-18.

Nicholls, J. G. (1975) "Causal attribution and other achievement-related cognitions: Effects of task outcome, attainment value and sex". Journal of Personality and Social Psychology, n. 31, p. 379-389.

Nunes, A. O. (2015) "Métodos matriciais e tensoriais como suporte à gestão nos Cursos de Engenharia e Sistemas e Mídias digitais na Universidade Federal do Ceará (UFC)". Tese (doutorado) - Universidade Federal do Ceará, Centro de Tecnologia, Programa de Pós-Graduação em Engenharia de Teleinformática, Fortaleza.

Ribeiro, G. O., Silva, T. E. V., Nunes, A. O., Pinto, F. A. P. e Vasconcelos, F. H. L. (2013) "Avaliação da Efetividade do Ensino em um Curso de Formação continuada Semipresencial”. Anais do XIX WIE.

Ribeiro, G. O., Silva, T. E. V., Nunes, A. O., Pinto, F. A. P. e Vasconcelos, F. H. L. (2014) "Perspectivas para a Redução da Evasão em EaD a partir da Avaliação da Qualidade do Ensino Online.” In: Anais do $3^{\circ}$ Congresso Brasileiro de Informática na Educação (CBIE 2014) e 20ª Workshop de Informática na Escola (WIE 2014), p.428-438.

Rocha, E. M., Joyce, C. R., Joyce, F. M. e Werlang, R. (2011) “A Automação da Avaliação Institucional na EAD”. Anais do XVII WIE.

Silva, T. E. V., Ribeiro, G. O., Nunes, A. O., Vasconcelos, F. H. L., e Andriola, W.B. (2015) "Avaliação dos Indicadores da Qualidade do Ensino Online: um estudo de caso". Anais dos Workshops do IV CBIE.

Sobrinho, J. D. (2011) "Avaliação institucional, instrumento da qualidade educativa: a experiência da Unicamp". In: Avaliação Institucional: teoria e experiências. José Dias Sobrinho, Newton César Balzan (orgs). $5^{a}$ ed. São Paulo: Cortez.

Urbina, N. A.; Pérez, C. B.; Rodríguez, H. R. (2013) "Aplicación del modelo ELQ em la evaluación de la calidad em la educación a distancia impartida em la UPPUEBLA”. RIED. Revista Iberoamericana de Educação à Distância, v.15, n.1, pp. 155-172. 